\title{
Packing patterns into words
}

\author{
Alexander Burstein \\ Department of Mathematics \\ Iowa State University \\ Ames, IA 50011-2064 USA \\ burstein@math. iastate.edu
}

\author{
Peter Hästö \\ Department of Mathematics \\ University of Michigan \\ Ann Arbor, MI 48105-1109 USA \\ peter.hasto@helsinki.fi
}

\author{
Toufik Mansour \\ Department of Mathematics \\ Haifa University \\ 31905 Haifa, Israel \\ toufik@math.haifa.ac.il
}

Submitted: May 29, 2003; Accepted: Nov 14, 2003; Published: Nov 20, 2003

MR Subject Classifications: Primary 05A15; Secondary 05A16

\begin{abstract}
In this article we generalize packing density problems from permutations to patterns with repeated letters and generalized patterns. We are able to find the packing density for some classes of patterns and several other short patterns.
\end{abstract}

The string 213322 contains three subsequences 233, 133, 122 each of which is orderisomorphic (or simply isomorphic) to the string 122, i.e. ordered in the same way as 122 . In this situation we call the string 122 a pattern.

Herb Wilf first proposed the systematic study of pattern containment in his 1992 address to the SIAM meeting on Discrete Mathematics. However, several earlier results on pattern containment exist, for example, those by Knuth [9] and Tarjan [15].

Most results on pattern containment actually deal with pattern avoidance, in other words, enumerate or consider properties of strings over a totally ordered alphabet which avoid a given pattern or set of patterns.

There is considerably less research on other aspects of pattern containment, specifically, on packing patterns into strings over a totally ordered alphabet (but see $[1,3,7,12$, 14]). In fact, all pattern packing except the one in [14] (later generalized in [1]) dealt with packing permutation patterns into permutations (i.e. strings without repeated letters). In this paper, we generalize the packing statistics and results to patterns over strings with repeated letters and relate them to the corresponding results on permutations. We deal 
with monotone and layered patterns in Section 2, with generalized patterns in Section 3, and with superpatterns (strings containing all patterns in a given set) in Section 4 .

\section{Preliminaries}

Let $[k]=\{1,2, \ldots, k\}$ be our canonical totally ordered alphabet on $k$ letters, and consider the set $[k]^{n}$ of $n$-letter words over $[k]$. We say that a pattern $\pi \in[l]^{m}$ occurs in $\sigma \in[k]^{n}$, or $\pi$ hits $\sigma$, or that $\sigma$ contains the pattern $\pi$, if there is a subsequence of $\sigma$ order-isomorphic to $\pi$.

Given a word $\sigma \in[k]^{n}$ and a set of patterns $\Pi \subseteq[l]^{m}$, let $\nu(\Pi, \sigma)$ be the total number of occurrences of patterns in $\Pi$ (П-patterns, for short) in $\sigma$. Obviously, the largest possible number of $\Pi$-occurrences in $\sigma$ is $\left(\begin{array}{l}n \\ m\end{array}\right)$, when each subsequence of length $m$ of $\sigma$ is an occurrence of a П-pattern. Define

$$
\begin{aligned}
\mu(\Pi, k, n) & =\max \left\{\nu(\Pi, \sigma) \mid \sigma \in[k]^{n}\right\}, \\
d(\Pi, \sigma) & =\frac{\nu(\Pi, \sigma)}{\left(\begin{array}{c}
n \\
m
\end{array}\right)} \text { and } \\
\delta(\Pi, k, n) & =\frac{\mu(\Pi, k, n)}{\left(\begin{array}{c}
n \\
m
\end{array}\right)}=\max \left\{d(\Pi, \sigma) \mid \sigma \in[k]^{n}\right\},
\end{aligned}
$$

respectively, the maximum number of $\Pi$-patterns in a word in $[k]^{n}$, the probability that a subsequence of $\sigma$ of length $m$ is an occurrence of a П-pattern, and the maximum such probability over words in $[k]^{n}$. We want to consider the asymptotic behavior of $\delta(\Pi, k, n)$ as $n \rightarrow \infty$ and $k \rightarrow \infty$.

Proposition 1.1 If $n>m$, then $\delta(\Pi, k, n) \leq \delta(\Pi, k, n-1)$ and $\delta(\Pi, k, n) \geq \delta(\Pi, k-1, n)$.

Proof. The proof of Proposition 1.1 in [1] also applies to the first inequality in our proposition, since possible repetition of letters is irrelevant here. To see that the second inequality is true, note that increasing $k$, i.e. allowing more letters in our alphabet, can only increase $\mu(\Pi, k, n)$, and hence $\delta(\Pi, k, n)$.

The greatest possible number of distinct letters in a word $\sigma$ of length $n$ is $n$, which implies that $\mu(\Pi, k, n)=\mu(\Pi, n, n)$ for $k \geq n$, and hence, $\delta(\Pi, k, n)=\delta(\Pi, n, n)$ for $k \geq n$. Therefore,

$$
\delta(\Pi, n, n)=\lim _{k \rightarrow \infty} \delta(\Pi, k, n) .
$$

We also have $\delta(\Pi, n, n)=\delta(\Pi, n+1, n) \geq \delta(\Pi, n+1, n+1)$, so $\delta(\Pi, n, n)$ is non-increasing and nonnegative, and there exists

$$
\delta(\Pi)=\lim _{n \rightarrow \infty} \delta(\Pi, n, n)=\lim _{n \rightarrow \infty} \lim _{k \rightarrow \infty} \delta(\Pi, k, n) .
$$

We call $\delta(\Pi)$ the packing density of $\Pi$. 
Obviously, there are two double limits. Since $\delta(\Pi, k, n)$ is non-increasing in $n$ and greater than 0 , it follows that

$$
\delta(\Pi, k)=\lim _{n \rightarrow \infty} \delta(\Pi, k, n) \in[0,1]
$$

exists and is nondecreasing in $k$. Hence, we can define

$$
\delta^{\prime}(\Pi)=\lim _{k \rightarrow \infty} \delta(\Pi, k)=\lim _{k \rightarrow \infty} \lim _{n \rightarrow \infty} \delta(\Pi, k, n) .
$$

It is easy to see that $\delta^{\prime}(\Pi) \leq \delta(\Pi)$. Naturally, one wishes to determine when $\delta^{\prime}(\Pi)=$ $\delta(\Pi)$. In this paper, we will provide one sufficient condition for this equality; however, we have not been able either to prove that $\delta^{\prime}(\Pi)=\delta(\Pi)$ for all $\Pi$ or to find $\Pi$ for which $\delta^{\prime}(\Pi)<\delta(\Pi)$.

The set $[k]^{n}$ is finite, so for each $k$ and $n$, there is a string $\sigma(\Pi, k, n) \in[k]^{n}$, not necessarily unique, such that $d(\Pi, \sigma(\Pi, k, n))=\delta(\Pi, k, n)$. To find $\delta(\Pi)$, we will need to find $\delta(\Pi, k, n)$, hence maximal $\Pi$-containing permutations $\sigma(\Pi, k, n)$ are of interest to us, especially, their asymptotic shape as $n \rightarrow \infty$ and $k \rightarrow \infty$.

Example 1.2 Let $\Pi=\left\{c_{m}\right\}$, where $c_{m}$ is a constant string of $m 1$ 's. Then, clearly, $\sigma(\Pi, k, n)=c_{n}$ and $d\left(c_{m}, c_{n}\right)=1$ for $n \geq m$, so $\delta\left(c_{m}, k, n\right)=1$ for $n \geq m$, and hence $\delta^{\prime}\left(c_{m}\right)=\delta\left(c_{m}\right)=1$ for any $m \geq 1$.

Example 1.3 Let $\Pi=\left\{i d_{m}\right\}$, where $i d_{m}$ is the identity permutation of $S_{m}$. Then $\sigma\left(i d_{m}, n, n\right)=i d_{n}$ and $d\left(i d_{m}, i d_{n}\right)=1$, so $\delta\left(i d_{m}, n, n\right)=1$ and $\delta\left(i d_{m}\right)=1$.

Determining $\delta^{\prime}\left(i d_{m}\right)$ is a bit harder. It is easy to see that $\sigma\left(i d_{m}, k, n\right)$ must be a nondecreasing string of digits in $[k]$. Let $n_{i}$ be the number of digits $i$ in $\sigma\left(i d_{m}, k, n\right)$, then $\mu\left(i d_{m}, k, n\right)=\nu\left(i d_{m}, \sigma\left(i d_{m}, k, n\right)\right)=n_{1} n_{2} \ldots n_{k}$ and $n_{1}+n_{2}+\cdots+n_{k}=n$. To maximize the above product we need $n_{1}=n_{2}=\cdots=n_{k}=\frac{n}{k}$. (More exactly, [12] shows that we should choose for $n_{i}$ 's to be such integers that $\left|n_{i}-\frac{n}{k}\right|<1$ and $\left|n_{1}+\cdots+n_{r}-\frac{r n}{k}\right|<1$ for each $r=1,2, \ldots, k$.) It follows that

$$
\delta\left(i d_{m}, k, n\right) \sim \frac{\left(\begin{array}{c}
k \\
m
\end{array}\right)\left(\frac{n}{k}\right)^{m}}{\left(\begin{array}{c}
n \\
m
\end{array}\right)}
$$

(where $a_{n} \sim b_{n}$ means $\lim _{n \rightarrow \infty} a_{n} / b_{n}=1$ ), so $\delta\left(i d_{m}, k\right)=\left(\begin{array}{c}k \\ m\end{array}\right) \frac{m !}{k^{m}}$, and thus $\delta^{\prime}\left(i d_{m}\right)=1$ as expected.

Packing density was initially defined for patterns in permutations. Therefore, we must show that the packing density on permutations agrees with the packing density on words.

Theorem 1.4 Let $\Pi \subseteq S_{m}$ be a set of permutation patterns, then

$$
\delta(\Pi)=\lim _{n \rightarrow \infty} \frac{\max \left\{\nu(\Pi, \sigma) \mid \sigma \in S_{n}\right\}}{\left(\begin{array}{c}
n \\
m
\end{array}\right)},
$$

i.e. the packing density of $\Pi$ on words is equal to that on permutations. 
Proof. It is enough to prove that

$$
\mu(\Pi, n, n)=\max \left\{\nu(\Pi, \sigma) \mid \sigma \in S_{n}\right\},
$$

in other words, that there is a permutation in $S_{n}$ among the maximal $\Pi$-containing words in $[n]^{n}$. Consider any maximal $\Pi$-containing word $\sigma \in[n]^{n}$. Let $n_{i}$ be the multiplicity of the letter $i$ in $\sigma$. Let $i_{j}$ denote the $j$ th occurrence of the letter $i$, and consider the map $f:[n]^{n} \rightarrow S_{n}$ induced by the map $i_{j} \mapsto\left(\sum_{r=1}^{i} n_{r}\right)-j+1$. Since all letters of each pattern in $\Pi$ are distinct, $\Pi$ occurs in $f(\sigma)$ at least at the same positions $\Pi$ occurs in $\sigma$, so $\nu(\Pi, f(\sigma)) \geq \nu(\Pi, \sigma)$. The rest is easy.

Apart from computing packing densities of patterns, we would also like to determine which patterns have equal packing densities, which ones are asymptotically more packable than others, etc. For example, it is easy to see that the packing density is invariant under the usual symmetry operations on $[l]^{m}$ : reversal $r: \tau(i) \rightarrow \tau(m-i+1)$ and complement $c: \tau(i) \rightarrow l-\tau(i)+1$, (packing density is also invariant under inverse $i: \tau \rightarrow \tau^{-1}$ when packing permutations into permutations). The operations $r$ and $c$ generate $D_{2}$, while $r, c, i$ generate $D_{4}$. Patterns which can be obtained from each other by a sequence of symmetry operations are said to belong to the same symmetry class.

Example 1.5 The symmetry class representatives of patterns in $[3]^{3}$ are 111, 112, 121, 123 and 132. We know that $\delta(111)=1=\delta(123)$. Galvin, Kleitmann and Stromquist (independently, unpublished, see chronology in [12]) showed that $\delta(132)=2 \sqrt{3}-3 \approx 0.4641$. Thus, we only need to determine the packing densities of 112 and 121 to completely classify patterns of length 3 .

Price [12] extended Stromquist's results [14] to packing a single pattern $\pi=1 m(m-$ 1) ...2 and handled other single patterns such as 2143 . Since we will also be concerned mostly with singleton sets of patterns $\Pi=\{\pi\}$, we will write $\delta(\pi)$ for $\delta(\{\pi\})$, etc.

Price's results deal with patterns of specific type, the so-called layered patterns.

Definition 1.6 A layered permutation is a strictly increasing sequence of strictly decreasing substrings. These substrings are called the layers of $\sigma$.

Notation 1.7 It easy to see that a layered permutation is uniquely determined by the sequence of its layer lengths, hence we may denote it by such sequence, e.g. $\widehat{321} \widehat{54} \widehat{9876}=$ $[3,2,4], \widehat{12} \widehat{3}=[1,1,1], \widehat{132}=[1,2], \widehat{21} \widehat{3}=[2,1], \widehat{321}=[3]$ are layered, with layers denoted by hats, while 312,231 are non-layered.

In fact, note that the union of symmetry classes of layered permutations consists of exactly the permutations avoiding patterns in the symmetry classes of 1342, 1423, 2413.

In [14], Stromquist proved a theorem (later generalized in [1]) on packing layered patterns into permutations. The inductive proof of this theorem defines a permutation (or a poset) $\pi$ to be layered on top (or LOT) if each of its maximal elements is greater than any non-maximal element. The set of these maximal elements is called the final layer of $\pi$ (even if $\pi$ is not necessarily layered). Recall that $\nu(\Pi, \sigma)$ is the total number of occurrences of patterns in $\Pi$ (П-patterns, for short) in a string $\sigma$. 
Proposition 1.8 Let $\Pi$ be a multiset of LOT permutations (not necessarily all distinct or of equal length). Then there is an LOT permutation $\sigma^{*} \in S_{n}$ which maximizes the expression

$$
\nu(\Pi, \sigma)=\sum_{\pi \in \Pi} a_{\pi} \nu(\pi, \sigma), \quad a_{\pi} \geq 0, \quad \sigma \in S_{n} .
$$

Furthermore, if the final layer of every $\pi \in \Pi$ has size greater than 1, then every such $\sigma^{*}$ is LOT.

Applying this proposition inductively, [1], following [14], obtains

Theorem 1.9 Let $\Pi$ be a multiset of layered permutations. Then there is a layered permutation $\sigma^{*}$ which maximizes the expression (1.1). Furthermore, if all the layers of every $\pi \in \Pi$ have size greater than 1 , then every such $\sigma^{*}$ is layered.

Following $[1,12]$, we will also define the $\ell$-layer packing density $\delta_{\ell}(\Pi)$ for sets of layered permutations $\Pi$ as the packing density of $\Pi$ among the permutations with at most $\ell$ layers. It was shown in both of the above works that $\delta(\Pi)=\lim _{\ell \rightarrow \infty} \delta_{\ell}(\Pi)$.

\section{Monotone and layered patterns}

The easiest type of patterns with repeated letters are those whose letters are nondecreasing (or non-increasing) from left to right. By analogy with layered patterns, we will consider nondecreasing patterns.

We will call a maximal constant segment of a word a block. For a letter $a$ and integer $k \geq 1$, we will define $a^{k}=\underbrace{a \ldots a}_{k}$.

Theorem 2.1 Let $\Pi \in[l]^{m}$ be a set of nondecreasing patterns $\pi=1^{a_{1}(\pi)} 2^{a_{2}(\pi)} \ldots l^{a_{l}(\pi)}$. For each $\pi \in \Pi \subseteq[l]^{m}$, let $\hat{\pi} \in S_{m}$ be the layered pattern $\hat{\pi}=\left[a_{1}(\pi), \ldots, a_{l}(\pi)\right]$, and let $\hat{\Pi}=\{\hat{\pi} \mid \pi \in \Pi\}$. Then $\delta(\Pi, k)=\delta_{k}(\hat{\Pi})$ and $\delta^{\prime}(\Pi)=\delta(\Pi)=\delta(\hat{\Pi})$.

PROOF. There is a natural bijection between nondecreasing patterns on $l$ letters and layered patterns with $l$ layers. The map $f$ of Theorem 1.4, induced by the map $i_{j} \mapsto$ $\left(\sum_{r=1}^{i} a_{r}(\pi)\right)-j+1$ (where $i_{j}$ is the $j$ th $i$ from the left), maps $\pi$ to $\hat{\pi}$. Clearly, $f^{-1}$ is induced by a map which takes each element in the $i$ th layer (the $i$ th basic subsequence, in general) to the integer $i$. (The $i$ th basic subsequence of a permutation consists of elements that are " $i$ " in some occurrence of pattern $i d_{i}$, but never " $i+1$ " in any occurrence of $i d_{i+1}$. For example, the first basic subsequence consists of the left-to-right minima.) Thus, occurrences of any $\pi \in \Pi$ in any word $\sigma$ correspond to occurrences of $\hat{\pi}$ in $f(\sigma)$, so $\nu(\Pi, \sigma)=\nu(\hat{\Pi}, f(\sigma))$. The rest is easy.

Example 2.2 Using the previous theorem and results of Price [12], we obtain $\delta(112)=$ $\delta(\widehat{21} \widehat{3})=2 \sqrt{3}-3, \delta(1122)=\delta(\widehat{21} \widehat{43})=3 / 8$. More generally, for $k \geq 2$,

$$
\delta(\underbrace{1 \ldots 1}_{k} 2)=k(1-\alpha) \alpha^{k-1}, \quad \text { where } 0<\alpha<1, \quad(1-k \alpha)^{k+1}=1-(k+1) \alpha .
$$


Similarly, for $r, s \geq 2$,

$$
\delta(\underbrace{1 \ldots 1}_{r} \underbrace{2 \ldots 2}_{s})=\delta(\underbrace{1 \ldots 1}_{r} \underbrace{2 \ldots 2}_{s}, 2)=\left(\begin{array}{c}
r+s \\
r
\end{array}\right) \frac{r^{r} s^{s}}{(r+s)^{r+s}} .
$$

In other words, the maximal packing is achieved on 2-letter words $11 \ldots 122 \ldots 2$ with strings of 1's and 2's of relative lengths $r /(r+s)$ and $s /(r+s)$. Using the results of Albert et al. [1], we also find that $\delta(1123)=\delta(1233)=\delta(1243)=3 / 8, \delta(\{122,112\})=$ $\delta(\{132,213\})=3 / 4$.

Notation 2.3 A monotone nondecreasing pattern is uniquely determined by the sequence of its block lengths. Because of this and as a consequence of Theorem 2.1, we may by abuse of notation denote a monotone nondecreasing pattern by the sequence of its block lengths, e.g. $112=[2,1], 122=[1,2], 123=[1,1,1]$.

By analogy with layered permutations, we define layered strings as follows.

Definition 2.4 A string $\pi \in[l]^{m}$ is layered if it is a concatenation of a strictly increasing sequence of non-increasing substrings. In other words, $\pi=\pi_{1} \ldots \pi_{r}$, where $\pi_{i}$ are nonincreasing, and $\pi_{1}<\cdots<\pi_{r}$ (that is any letter of $\pi_{i}$ is less than any letter of $\pi_{j}$ if $i \leq j$ ). Substrings $\pi_{i}$ maximal with respect to these properties are called the layers of $\pi$.

Definition 2.5 Let us say that the layered permutation $\pi$ is simple if there exists a sequence $\left\{\sigma_{n}\right\}$ of layered permutations with $\sigma_{n} \in S_{n}$ such that every $\sigma_{n}$ has $r$ layers and $\lim _{n \rightarrow \infty} d\left(\pi, \sigma_{n}\right)=\delta(\pi)$.

Simple permutations are, as indicated by the name, the easiest type of permutations to calculate the packing density of. Indeed, it was shown in [7, Theorem 1.2] that the layered permutation $\pi$ of type $\left[m_{1}, \ldots, m_{r}\right]$ with $\log _{2}(r+1) \leq \min \left\{m_{i}\right\}$ is simple and that in this case

$$
d(\pi)=\frac{m !}{m^{m}} \prod_{k=1}^{r} \frac{m_{k}^{m_{k}}}{m_{k} !}
$$

where $m:=m_{1}+\ldots+m_{r}$.

Theorem 2.6 Let $\pi$ be layered pattern with each layer isomorphic to either $k \ldots 1$ or $1 \ldots 1$. Let $\pi^{\prime}$ be the layered permutation with layer lengths equal to those of $\pi$. If $\pi$ is simple, then $\delta(\pi)=\delta\left(\pi^{\prime}\right)$.

Proof. Let us denote by $m$ the number of layers in $\pi$. If $f$ is an operation as in Theorems 1.4 and 2.1 and $\pi$ is layered, then $\pi^{\prime}=f(\pi)$ is layered. Since $\pi^{\prime}$ is simple, the $\pi^{\prime}$-maximal permutation is essentially one with $m$ layers of size proportional to those of $\pi$. But transforming this permutation into a layered pattern by changing a layer to block if the corresponding layer of $\pi$ is a block gives a pattern $\sigma$ for which $d(\pi, \sigma) \rightarrow \delta\left(\pi^{\prime}\right)$. Therefore $\delta(\pi) \geq \delta\left(\pi^{\prime}\right)$.

Let $\sigma$ be a $\pi$-maximal pattern. Then every occurence of $\pi$ in $\sigma$ is an occurence of $f(\pi)=\pi^{\prime}$ in $f(\sigma)$, so that $\delta(\pi) \leq \delta\left(\pi^{\prime}\right)$. It follows that $\delta(\pi)=\delta\left(\pi^{\prime}\right)$. 
Example 2.7 Let $\pi=k(k-1) \ldots 1(k+1)^{q}$. If $q \geq 2$ then

$$
\delta(\pi)=\left(\begin{array}{c}
k+q \\
k
\end{array}\right) \frac{k^{k} q^{q}}{(k+q)^{k+q}} .
$$

If $q=1$ then $\delta(\pi)=\delta\left(1^{k} 2\right)=\delta([k, 1])$, given in Example 2.2. The first claim follows by Theorem 2.6 and [7, Theorem 1.2]. The second claim follows by Theorem 2.6 and [12, Theorem 5.2].

Conjecture 2.8 If $\Pi$ is a set of layered patterns, then $\delta^{\prime}(\Pi)=\delta(\Pi)$ and among maximal I-containing strings in $[k]^{n}$, there is one which is layered.

Next we will discuss a non-monotone type of patterns related to monotone patterns.

Theorem 2.9 Let $\pi=1^{p} 2^{r} 1^{q}$, for $p, q, r \geq 1$. Then

$$
\delta(\pi)=\left(\begin{array}{c}
p+q \\
p
\end{array}\right) \frac{p^{p} q^{q}}{(p+q)^{p+q}} \delta\left(1^{p+q} 2^{r}\right)=\left(\begin{array}{c}
p+q \\
p
\end{array}\right) \frac{p^{p} q^{q}}{(p+q)^{p+q}} \delta([p+q, r]) .
$$

Proof. Let $\sigma$ be a $\pi$-maximal pattern of length $n$. Denote by $a_{i}$ the number of $i$ 's in $\sigma$. It is clear that $\sigma$ can be assumed to have at least two blocks at every height except the greatest.

Let us compare the hits (occurrences) of $\pi$ in $\sigma$ with those of the pattern $\pi^{\prime}=1^{p+q} 2^{r}$ in $\sigma^{\prime}=1^{a_{1}} 2^{a_{2}} \ldots k^{a_{k}}$. Let us count the number of hits in each case with the blocks of 1 's at height $i$ and the 2's at height $j>i$. The maximum number of such hits of $\pi$ in $\sigma$ occurs in the pattern $i^{p a_{i} /(p+q)} j^{a_{j}} i^{q a_{i} /(p+q)}$ and equals

$$
\left(\begin{array}{c}
p a_{i} /(p+q) \\
p
\end{array}\right)\left(\begin{array}{c}
a_{j} \\
r
\end{array}\right)\left(\begin{array}{c}
q a_{i} /(p+q) \\
q
\end{array}\right) .
$$

(This argument is strictly true only if $a_{i}$ is divisible by $p+q$, otherwise we have to round suitably.) On the other hand the hits of $\pi^{\prime}$ in $\sigma^{\prime}$ with the 1's and the 2's at these heights occurs in

$$
\left(\begin{array}{c}
a_{i} \\
p+q
\end{array}\right)\left(\begin{array}{c}
a_{j} \\
r
\end{array}\right)
$$

cases. By considering this ratio for large $a_{i}$, we find that

$$
\nu(\pi, \sigma) \leq\left(\begin{array}{c}
p+q \\
p
\end{array}\right) \frac{p^{p} q^{q}}{(p+q)^{p+q}} \nu\left(\pi^{\prime}, \sigma^{\prime}\right) .
$$

(We do not need to consider small $a_{i}$ 's since their contribution as $n \rightarrow \infty$ will be negligible.) But we know the density of $\pi^{\prime}$ by Theorem 2.1, and so it follows that

$$
\delta(\pi) \leq\left(\begin{array}{c}
p+q \\
p
\end{array}\right) \frac{p^{p} q^{q}}{(p+q)^{p+q}} \delta([p+q, r]) .
$$

On the other hand it is easy to see that we can construct patterns containing this many $\pi$ 's; we take a $\pi^{\prime}$-maximal pattern and split each block except the one on the highest level 
into two blocks of relative sizes $p$ and $q$ and place the first before and the latter after all higher height blocks. Therefore the inequality is in fact is an equality, and the theorem is proved.

Note that Theorem 2.9 also applies when $p=0$ or $q=0$, in which case it reduces to an identity.

Remark 2.10 If $r>1$ in the previous theorem, then

$$
\delta([p+q, r])=\left(\begin{array}{c}
p+q+r \\
r
\end{array}\right) \frac{(p+q)^{p+q} r^{r}}{(p+q+r)^{p+q+r}},
$$

and so

$$
\delta(\pi)=\left(\begin{array}{c}
p+q+r \\
p, q, r
\end{array}\right) \frac{p^{p} q^{q} r^{r}}{(p+q+r)^{p+q+r}} .
$$

The $\pi$-maximizing string here is of the type $1^{a} 2^{c} 1^{b}$ with asymptotic layer lengths

$$
\left(\frac{p}{p+q+r}, \frac{r}{p+q+r}, \frac{q}{p+q+r}\right) .
$$

Remark 2.11 When $r=1$, we can calculate $\delta([p+q, r])$ as in Example 2.2, which yields

$$
\delta(\pi)=\left(\begin{array}{c}
p+q \\
p
\end{array}\right) p^{p} q^{q}(1-(p+q) \alpha) \alpha^{p+q-1},
$$

where $\alpha \in(0,1)$ is the unique solution of $(1-s x)^{s+1}=1-(s+1) x$ and $s=p+q$. The $\pi$-maximizing string here is of the type $1^{a_{1}} 2^{a_{2}} 3^{a_{3}} \ldots \ldots 3^{b_{3}} 2^{b_{2}} 1^{b_{1}}$ with asymptotic layer lengths $\left(p \alpha, p \alpha(1-s \alpha), p \alpha(1-s \alpha)^{2}, \ldots, \ldots, q \alpha(1-s \alpha)^{2}, q \alpha(1-s \alpha), q \alpha\right)$.

As an aside we note that

$$
\alpha=\frac{1}{s+1}-(s+1)^{-(s+2)}+O\left((s+1)^{-2 s}\right),
$$

since for $x_{0}=1 /(s+1)-(s+1)^{-(s+2)}$ we have

$$
\begin{aligned}
\left(1-s x_{0}\right)^{s+1}+(s+1) x_{0}-1 & =\left(\frac{1}{s+1}+\frac{s}{(s+1)^{s+2}}\right)^{s+1}-(s+1)^{-(s+1)} \\
& =\frac{s}{(s+1)^{2 s+1}}+O\left((s+1)^{1-3 s}\right)
\end{aligned}
$$

For $s \geq 3$, the error in $\alpha$ is at most $4^{-6}<0.00025$, so $x_{0}$ approximates $\alpha$ up to at least 3 decimal places.

Example 2.12 $\delta(121)=\frac{1}{2} \delta(112)=\frac{1}{2} \delta(213)=\sqrt{3}-3 / 2$. This completes the inventory of packing densities of 3-letter patterns by symmetry class.

\begin{tabular}{|c||c|c|c|c|c|}
\hline Symmetry class & 111 & 112 & 121 & 123 & 132 \\
\hline Packing density & 1 & $2 \sqrt{3}-3$ & $\frac{2 \sqrt{3}-3}{2}$ & 1 & $2 \sqrt{3}-3$ \\
\hline
\end{tabular}




\section{Generalized patterns}

Here we consider packing generalized patterns into words. Generalized patterns were introduced by Babson and Steingrímsson [2] and allow the requirement that some adjacent letters in a pattern be adjacent in its occurrences in an ambient string as well. For example, an occurrence of a generalized pattern 21-3 in a permutation $\pi=a_{1} a_{2} \cdots a_{n}$ is a subsequence $a_{i} a_{i+1} a_{j}$ of $\pi$ such that $a_{i+1}<a_{i}<a_{j}$. Clearly, in the new notation, classical patterns are those with all hyphens, such as 1-3-2.

Notation 3.1 This notation (introduced in [2]) may be a little confusing since classical patterns (the ones with all hyphens) were previously written the same way as the generalized patterns with all adjacent letters (i.e. with no hyphens). From now on, we will use the generalized pattern notation. However, if we consider subword patterns (those with no hyphens), we may write $\pi_{\mathrm{g}}$ for a generalized pattern $\pi$ without hyphens where the context allows for ambiguity.

If $\pi \in[l]^{m}$ is a generalized pattern with $b$ blocks of consecutive letters (i.e. $b-1$ hyphens), then it is easy to see by considering the positions of the first letters of the blocks of $\pi$ that the largest possible number of times $\pi$ can occur in $\sigma \in[k]^{n}$ is

$$
\left(\begin{array}{c}
n-m+b \\
b
\end{array}\right)
$$

(this yields $\left(\begin{array}{c}n \\ m\end{array}\right)$ when $b=m$, i.e. when $\pi$ is a classical pattern).

In fact, this maximum is achieved when $\pi$ is a constant generalized pattern, i.e. any of the generalized patterns obtained from the constant strings $11 \ldots 1$ by inserting hyphens at arbitrary positions (possibly, none). Obviously, maximal $\pi$-containing strings are the constant strings of length $n$. Thus, any set of constant generalized patterns has packing density 1. Similarly, any set $\Pi$ of hyphenated identity generalized patterns has $\delta(\Pi)=1$.

Given a set of generalized patterns with $b$ blocks, $\Pi \subseteq[l]^{m}$, we define the packing density of $\Pi$ similarly to that of a set of classical patterns. We will use the same notation as in Section 1 for the generalized patterns.

It is not difficult to see that the analog of Theorem 1.4 holds for generalized patterns as well.

Theorem 3.2 Let $\Pi \subseteq S_{m}$ be a set of generalized permutation patterns. The packing density of $\Pi$ on words is equal to that on permutations.

Proof. The same argument as in Theorem 1.4 shows that among maximal $\Pi$-containing strings in $[n]^{n}$ there is one which has no repeated letters.

\subsection{Generalized patterns without hyphens}

Theorem 3.3 Let $\pi=1^{a_{1}} 2^{a_{2}} \ldots l^{a_{l}} \in[l]^{m}(l>1)$ be a nonconstant monotone generalized pattern without hyphens. If there exists a positive integer $j \leq l-2$ such that $a_{1} \leq a_{j+1}$, 
$a_{i}=a_{i+j}(2 \leq i \leq l-j-1)$ and $a_{l-j} \geq a_{l}$, then we denote by $j_{0}$ the least such $j$ and define $M_{\pi}=a_{2}+\cdots+a_{j_{0}+1}$. Otherwise we set $M_{\pi}=\max \left\{a_{1}, a_{l}\right\}+a_{2}+\ldots+a_{l-1}$. In either case we have $\delta(\pi)=\delta^{\prime}(\pi)=1 / M_{\pi}$.

Proof. $M_{\pi}$ is the smallest shift at which $\pi$ overlaps with itself. The rest is clear.

Theorem 3.4 Let $\pi=\left[a_{1}, a_{2}, \ldots, a_{l}\right] \in S_{m}$ be any l-layer $(l>1)$ generalized pattern without hyphens. Let $M_{\pi}$ be as in Theorem 3.3. Then $\delta(\pi)=\delta^{\prime}(\pi)=1 / M_{\pi}$.

Proof. The same mapping as in Theorem 2.1 shows that our $\pi$ has the same packing density as the corresponding monotone generalized pattern without hyphens of Theorem 3.3 .

Corollary 3.5 Let $\pi_{1}=11 \ldots 12_{g} \in[2]^{m}$ and $\pi_{2}=1 m(m-1) \ldots 2_{g} \in[m]^{m}$, then $\delta\left(\pi_{1}\right)=\delta^{\prime}\left(\pi_{1}\right)=1 /(m-1)$ and $\delta\left(\pi_{2}\right)=\delta^{\prime}\left(\pi_{2}\right)=1 /(m-1)$.

For instance, $\delta\left(112_{g}\right)=\delta^{\prime}\left(112_{g}\right)=1 / 2, \delta\left(132_{g}\right)=\delta^{\prime}\left(132_{g}\right)=1 / 2$ and $\delta\left(123_{g}\right)=$ $\delta^{\prime}\left(123_{g}\right)=1$.

\subsection{Generalized patterns with one hyphen}

The maximal number of occurrences of a generalized pattern in $[l]^{m}$ with one hyphen (i.e. with $b=2$ blocks) is $\left(\begin{array}{c}n-m+2 \\ 2\end{array}\right) \sim n^{2} / 2$ as $n \rightarrow \infty$.

Proposition 3.6 $\delta(11-2)=\delta^{\prime}(11-2)=1$.

Proof. Let $\sigma \in[k]^{n}$ be a maximal (11-2)-containing word, then $\sigma$ is a monotone nondecreasing string in which the letter $i$ occurs $n_{i}$ times, $n_{1}+\cdots+n_{k}=n$. Then

$$
\mu(11-2, n, k)=\max \left\{\sum_{i=1}^{k}\left(n_{i}-1\right)\left(n_{i+1}+\cdots+n_{k}\right): n_{1}+\cdots+n_{k}=n\right\} .
$$

From here, it is not difficult to determine that $\mu(11-2, k, n) \sim n^{2} / 2$ as $n \rightarrow \infty$. Choose $n_{i}$ 's to be such integers that $\left|n_{i}-\frac{n}{k}\right|<1$ and $\left|n_{1}+\cdots+n_{r}-\frac{r n}{k}\right|<1$ for each $r=1,2, \ldots, k$. Then

$$
\mu(11-2, n, k) \sim\left(\frac{n}{k}\right)^{2}\left(\begin{array}{l}
k \\
2
\end{array}\right),
$$

out of $\left(\begin{array}{c}n-1 \\ 2\end{array}\right)$ maximum possible occurrences, and the result follows.

Proposition $3.7 \delta(12-3)=\delta(21-3)=1$.

Proof. For pattern 12-3, consider the identity permutation. For pattern 21-3, consider the layered permutations of length $n$ with $\sqrt{n}$ layers of length $\sqrt{n}$.

We think, but have not been able to prove rigorously, that $\delta(12-1)=\delta^{\prime}(12-1)=1 / 3$. At least $\delta(12-1,2)=1 / 3$, since in this case the string with the maximal number of occurrences of $12-1$ is of the type

$$
\sigma=1212 \cdots 1211 . .1 \in[2]^{n}
$$


where the string 12 occurs in $\sigma$ exactly $d$ times. So

$$
\mu(12-1, n, 2)=\max _{1 \leq d \leq n}(d(d-1) / 2+d(n-2 d))
$$

and the maximum occurs at $d \sim n / 3$. It seems that allowing more symbols in $\sigma$ does not change anything, but here we could not find a proof.

A more general question related to this and somewhat analogous to the question of simple layered permutations is: for which $\pi \in[k]^{n}$ is $\delta(\pi, k)=\delta(\pi)$ ?

\section{The problem of the shortest superpattern}

The problem of the shortest superpattern problem deals with packing different patterns into a word. It was treated in [5] in the case of permutation patterns. Here we consider the analogous problem in which the patterns can contain repeated letters.

Let $n(l, m)$ be the length of the shortest superpattern for $[l]^{m}$, i.e. the shortest word that contains every pattern of length $m$ on at most $l$ letters. Clearly, $n(l, m)=n(m, m)$ for $m \leq l$, hence we are interested only in the values of $n(l, m)$ for $m \geq l$.

For example, $n(2,2)=3$ (since 121 contains patterns $11,12,21)$ and $n(3,3)=7$ (since 3123132 contains patterns $111,112,121,211,122,212,221,123,132,213,231,312,321)$. The following construction for a superpattern for $[l]^{l}$ is given in [10].

Lemma 4.1 For any $l \geq 3, n(l, l) \leq l^{2}-2 l+4$.

ProOF. Consider the word $\bar{\tau}=\left(i d_{l-1}\right)^{l-2} 12$ where $i d_{l}=123 \ldots l$. Now insert $l$ copies of letter $l$ as follows. Insert the first $l$ at the beginning of $\bar{\tau}$, then, for $i=1,2, \ldots, l-1$, insert the $(i+1)$-st copy of $l$ immediately after the $i$ th copy of $l-i$. The string $\tau$ constructed thus has the length $(l-1)(l-2)+2+l=l^{2}-2 l+4$. It is easy to check that $\tau$ is a superpattern for $[l]^{l}$.

Example 4.2 For instance, for $l=5$ we get $\bar{\tau}=12341234123412$, and therefore $\tau=$ $\underline{5} 1234 \underline{5} 123 \underline{5} 412 \underline{5} 341 \underline{5} 2$.

A straightforward corollary of Lemma 4.1 is the following.

Corollary 4.3 For any $m \geq l \geq 3, n(m, l) \leq(m-2) l+4$.

ProOf. The concatenation of $\tau$ from Lemma 4.1 and $\left(i d_{l}\right)^{m-l}$ is a superpattern for $[l]^{m}$.

The upper bound in Corollary 4.3 can probably be improved. However, in the case of $n(l, l)$, it is a long-standing conjecture [11] (also mentioned in [6]) that the upper bound is apparently a lower bound as well.

Conjecture 4.4 For any $l \geq 3, n(l, l)=l^{2}-2 l+4$. 
The difficulty of the conjecture is apparent given that the best known lower bound for $n(l, l)$ is

$$
n(l, l) \geq n^{2}-c n^{7 / 4+\epsilon}
$$

for any $\epsilon>0$ and some constant $c=c(\epsilon)$ (see [11]).

This differs from the corresponding result in [5] on permutation patterns, i.e. those in $S_{l}$, where the upper bound of $3 l^{2} / 4$ for the length of the shortest common superpattern was established, and there is numerical evidence that the actual value is closer to $l^{2} / 2$.

Acknowledgement. The authors are grateful to Sergey Kitaev who brought the results in $[6,8,10,11]$ to their attention.

\section{References}

[1] M.H. Albert, M.D. Atkinson, C.C. Handley, D.A. Holton, W. Stromquist, On packing densities of permutations, Electron. J. Combin. 9 (2002), \#R5.

[2] E. Babson, E. Steingrímsson, Generalized permutation patterns and a classification of the Mahonian statistics, Sém. Lothar. Combin., B44b:18pp, 2000.

[3] M. Bóna, B.E. Sagan, V.R. Vatter, Pattern frequency sequences and internal zeros, Adv. in Appl. Math. 28 (2002), 395-420.

[4] A. Burstein, Enumeration of words with forbidden patterns, Ph.D. thesis, University of Pennsylvania, 1998.

[5] H. Eriksson, K. Eriksson, S. Linusson, J. Wästlund, Dense packing of patterns in a permutation, Proceedings of the 15th Conference on Formal Power Series and Algenbraic Combinatorics, Melbourne, Australia, 8-12 July 2002, paper \#26.

[6] A.A. Evdokimov, V.V. Nyu, Length of a supersequence for a set of binary words with a given number of units, Metody Diskretnogo Analiza, 52 (1992), 49-58, in Russian.

[7] P.A. Hästö, The packing density of other layered permutations, Electron. J. Combin. 9(2) (2002), \#R1.

[8] D.J. Kleitman, D.J. Kwiatkowski, A lower bound on the length of a sequence containing all permutations as subsequences. J. Combin. Theory Ser. A 21 (1976), no. $2,129-136$.

[9] D.E. Knuth, The Art of Computer Programming, vols. 1, 3, Addison-Wesley, NY, 1968, 1973.

[10] M.L. Kontsevich, Uniform placements, Kvant 7 (1985), 51-52, in Russian.

[11] D. Maier, The complexity of some problems on subsequences and supersequences, $J$. Association for Computing Machinery 25 (1978), no. 2, 322-336. 
[12] A. Price, Packing densities of layered patterns, Ph.D. thesis, University of Pennsylvania, 1997.

[13] R. Simion, F.W. Schmidt, Restricted Permutations, European J. Combin. 6 (1985), $383-406$.

[14] W. Stromquist, Packing layered posets into posets, manuscript, unpublished, 1993.

[15] R. Tarjan, Sorting using networks of queues and stacks, J. Association for Computing Machinery 19 (1972), 341-346. 